\title{
GLAD!
}

Revue sur le langage, le genre, les sexualités

$02 \mid 2017$

Varia

\section{Les enjeux idéologiques et argumentatifs de la formule "same sex marriage »}

Un phénomène de lexicologie socio-politique

Going beyond the Lexicological Use of "Same Sex Marriage": towards a

Qualitative Corpus-Based Analysis of Ideology and Argumentative Strategies at

Stake

\section{François Labatut}

\section{(2) OpenEdition}

Journals

Édition électronique

URL : http://journals.openedition.org/glad/580

DOI : $10.4000 /$ glad.580

ISSN : 2551-0819

Éditeur

Association GSL

Référence électronique

François Labatut, "Les enjeux idéologiques et argumentatifs de la formule "same sex marriage » , GLAD! [En ligne], 02 | 2017, mis en ligne le 01 juin 2017, consulté le 21 janvier 2021. URL : http:// journals.openedition.org/glad/580; DOI : https://doi.org/10.4000/glad.580

Ce document a été généré automatiquement le 21 janvier 2021.

\section{(c) $(1)$}

La revue GLAD! est mise à disposition selon les termes de la Licence Creative Commons Attribution -

Pas d'Utilisation Commerciale - Pas de Modification 4.0 International. 


\section{Les enjeux idéologiques et argumentatifs de la formule «same sex marriage »}

Un phénomène de lexicologie socio-politique

Going beyond the Lexicological Use of "Same Sex Marriage": towards a

Qualitative Corpus-Based Analysis of Ideology and Argumentative Strategies at

Stake

François Labatut

\section{L'ouverture du mariage aux couples de même sexe : des enjeux de formulation}

1 Le 17 juillet 2013, le Royaume-Uni adopte la loi intitulée Marriage (Same Sex Couples) Act. Cette dernière, en rendant justiciables les couples de même sexe au mariage, se présente comme une étape symbolique majeure dans la lutte pour la reconnaissance des droits LGBTQ+ dans le pays. Elle s'inscrit ainsi dans la lignée de lois telles que le Sexual Offences Act de 1967, qui décriminalise les rapports sexuels entre personnes de même sexe de plus de vingt et un ans, et le Civil Partnership Act de 2004, qui octroie aux couples de même sexe des avantages similaires à ceux du mariage civil.

2 Les couples de même sexe et les couples de sexe opposé sont dorénavant représentés aux yeux de la loi comme symboliquement égaux.

3 Mais qu'en est-il cependant à l'échelle linguistique? Que renferme et symbolise l'inscription en langue de l'expression « same sex marriage » par rapport aux expressions déjà existantes telles que "gay marriage » et «homosexual marriage "? Quelles en sont les implications idéologiques? Comment participe-t-elle de la construction discursive de cet idéal égalitaire représenté par le Marriage (Same Sex Couples) Act à l'échelle de la société? 
Cet article tente d'apporter quelques éléments de réponse à partir de l'étude discursive de l'expression "same sex marriage », sous le prisme de la notion de formule, en prenant appui sur les phénomènes collocationnels significatifs d'un point de vue statistique présents dans le corpus d'étude élaboré spécifiquement pour ce projet.

5 Pour ce faire, après avoir présenté dans un premier temps le corpus d'étude et la méthodologie d'analyse utilisés, j'expliciterai les parallèles possibles entre la lexie complexe "same sex marriage » et la notion de formule. L'étude systématique des phénomènes collocationnels rendra compte des différences sémantiques et discursives, et par extension socio-cognitives, de cette expression par rapport aux deux autres formules statistiquement concurrentes présentes dans mes données, " gay marriage » et "homosexual marriage», en vue de montrer la spécialisation lexicale de chacune de ces trois formules et leurs spécificités rhétoriques.

\section{Corpus d'étude : combiner débats parlementaires et associated memoranda}

6 Le corpus d'étude se compose de l'ensemble des ressources textuelles disponibles sur le site internet du parlement britannique ${ }^{1}$ relatif aux débats sur l'adoption du projet de loi same sex marriage.

7 Deux sources principales ont ainsi pu être recueillies: la totalité des transcriptions officielles des débats qui se sont déroulés du 12 février 2013 au 12 mars 2013, et l'ensemble des associated memoranda. Ces derniers correspondent à des témoignages écrits produits par la société civile et diffusés lors des débats dans l'enceinte parlementaire afin d'apporter aux parlementaires des arguments supplémentaires et des éclairages nouveaux sur les problématiques abordées.

8 Suite au nettoyage, à l'uniformisation et au changement de format (.txt) des données afin de les rendre analysables par le logiciel Wmatrix, trois sous-corpus ont été élaborés: un premier, qui regroupe l'ensemble des transcriptions des débats parlementaires (appelé maintenant Corpus-Débat; +250 000 mots), et deux autres, composés des associated memoranda. Ces derniers ont étés organisés en deux autres corpus selon qu'ils étaient en faveur (appelé maintenant Discours-PRO; +60 000 mots) ou contre (appelé maintenant Discours-ANTI ; +90000 mots) le projet de loi.

9 La distinction entre les associated memoranda favorables ou, au contraire, opposés à l'ouverture du mariage aux couples de même sexe a été effectuée après lecture de chaque mémorandum. Le processus de séparation s'est appuyé sur les normes rédactionnelles des associated memoranda. En effet, ces dernières préconisent d'expliciter clairement dès le début de chaque mémoire le point de vue défendu par ce dernier, ce qui a grandement facilité le travail de tri. 


\section{Spécificités et orientation méthodologique et théorique de l'étude}

\section{Linguistique de corpus : les collocations, des marqueurs de discours significatifs}

Hoey (2005) a démontré en quoi les associations privilégiées entre lexèmes, c'est-à-dire les collocations, sont le marqueur d'une connaissance inconsciente de la langue partagée entre les membres d'une communauté donnée. Les collocations ne peuvent être réduites à un unique phénomène syntaxique de répétition et de préférences lexicales. Au contraire, elles traduisent ce que Baker a appelé des "associations inconscientes " (2006: 116) qui sont nécessaires à la construction d'un discours et au maintien de sa cohérence. À ce sujet, les travaux de Halliday et Hasan (1976) ont souligné l'importance des phénomènes collocationnels dans le sentiment d'unification instancié par la cohésion lexicale interne d'un texte.

Par ailleurs, comme l'a indiqué Stubbs (2001: 215), les collocations, parce qu'elles établissent un rapport entre sémantique et syntaxe, ont un rôle prépondérant dans la configuration de la prosodie sémantique propre à un discours, c'est-à-dire les phénomènes de connotations et d'évaluation qui sont impliqués par l'utilisation d'un terme à la place d'un autre. Par exemple, des différences considérables en termes de connotations et d'évaluations, positives ou négatives, entre les lexèmes "gay » et " homosexual », apparaissent dans mes corpus. Et ce sont les collocations qui permettent d'en souligner les particularités et le fonctionnement à l'échelle non plus du lexème mais du discours, qu'il soit pour ou contre la légalisation du mariage pour les couples de même sexe.

\section{Wmatrix : pour un traitement quantitatif original des collocations ${ }^{2}$}

12 Le traitement des phénomènes collocationnels et des calculs statistiques ${ }^{3}$ a été effectué à l'aide du logiciel en ligne Wmatrix ${ }^{4}$. Afin de faire ressortir les collocations les plus significatives, chacun des trois corpus a fait l'objet de comparaison avec un autre corpus de taille équivalente. Ainsi, le Corpus-Débat a été confronté avec le BNC Sampler CG Institutional, corpus de référence intégré à Wmatrix. Le Discours-PRO et le DiscoursANTI ont été comparés entre eux, afin de faire ressortir plus clairement les préférences lexicales et sémantiques de ces deux discours antagonistes.

13 A été ainsi identifié l'ensemble des principaux schémas collocationnels significatifs dans les trois corpus contenant les lexèmes "marriage ", "same sex», "gay» et "homosexual ». Enfin, chaque phénomène a été importé dans des tableaux Excel pour classification et annotations complémentaires; n'ont été retenus que les résultats en relation étroite avec et/ou faisant référence à la notion de mariage.

Seuls les phénomènes significatifs d'un point de vue statistique ont été sélectionnés, c'est-à-dire dont la récurrence systématique dans l'un des corpus étudiés ne peut pas être due au hasard mais participe à son échelle à la cohérence du discours dans lequel le phénomène s'inscrit. L'avantage de cet ancrage théorique et méthodologique est qu'il ouvre l'accès à des corpus de taille très conséquente, comme mon corpus d'étude, qu'une étude qualitative ne permettrait pas d'embrasser. Néanmoins, ce choix 
méthodologique entraîne la non-prise en considération dans mes résultats de phénomènes collocationnels certes présents dans mes corpus, mais qui ne ressortent pas comme statistiquement significatifs. C'est le cas notamment des lemmes «lesbian.s» et "transsexual.s» qui, bien qu'apparaissant respectivement 40 fois et 16 fois dans le Discours-PRO, et 11 fois et 0 fois dans le Discours-ANTI, n'obtiennent dans aucun des cas un rapport de vraisemblance supérieur à la limite précédemment fixée de $15.13^{5}$.

\section{L'éclairage de l'analyse du discours : la notion de formule}

De nombreux travaux en analyse du discours ont mis en relation les phénomènes de collocations avec ceux de figements discursifs (Tutin 2002 et 2012), et ce en particulier à travers la notion de formule. À la suite d'Alice Krieg-Planque, la formule renvoie à « un ensemble de formulations qui, du fait de leurs emplois à un moment donné et dans un espace public donné, cristallisent des enjeux politiques et sociaux que ces expressions contribuent dans le même temps à construire » (2009: 7). Cette définition s'applique avec aisance à la lexie complexe «same sex marriage». En effet, le recours à la formule "same sex marriage " devient nécessaire pour quiconque souhaite prendre part au débat, qu'il soit pour ou contre ledit projet de loi. La formule devient ainsi un passage obligé. Et c'est à partir de cet axe central que vont s'organiser les discours antagonistes émergents. La formule se retrouve au cœur du débat et cristallise en conséquence les enjeux politiques sous-jacents à son utilisation : l'ouverture ou non de l'institution du mariage aux couples de même sexe. Par ailleurs, l'on se situe à un moment précis: le temps consacré aux débats et à l'échange d'idées relatifs à l'adoption ou non dudit projet de loi. L'espace public est clairement délimité, en ce qu'il se restreint aux différents comités constitués à cette occasion. Enfin, la cristallisation des enjeux politiques et sociaux s'opère dans la structure lexicale même de la formule, qui traduit en quelque sorte un résumé de l'enjeu final du projet de loi: la reconnaissance juridique des couples de même sexe.

\section{Inscription en langue et créativité linguistique : de l'origine lexicogénique de « same sex marriage »}

D'un point de vue morphologique, la formule «same sex marriage » découle du processus appelé composition, qui se caractérise par la «conjonction lexicale de deux bases autonomes » (Paillard $2000:$ 45). Véritable « micro syntaxe», selon la terminologie de Benveniste, la composition permet l'association syntaxique de deux « notions abstraites de toute actualisation» (1974: 145). La tête de la locution «same sex marriage» est le nom commun «marriage». C'est un cas de composition dite endocentrique de type germanique, c'est-à-dire une composition où l'élément qui impose à la fois la catégorie lexicale et le sens principal de l'ensemble est situé à l'extrémité droite ; le modificateur du nom, ici "same sex", se positionne à sa gauche, respectant ainsi l'ordre de composition et d'organisation syntaxique des énoncés en anglais. Il est par ailleurs utile de mentionner que le modificateur "same sex" est lui-même issu d'un processus de composition adjectivale. 
Les propriétés discursives de "same sex marriage » : figement, discursivité, référent social et dimension polémique

17 À partir des précédents travaux de Marianne Ebel et Pierre Fiala (1977), Alice KriegPlanque propose une grille d'analyse qui met en exergue quatre propriétés formelles et fondamentales de la formule.

Principalement, le phénomène se traduit par l'inscription de la formule au niveau du discours. Bien qu'ancrée dans le domaine linguistique, comme l'a montré l'étude du profil lexicogénique de "same sex marriage ", la formule est à appréhender avant tout comme un phénomène discursif : c'est une séquence stabilisée dont la particularité, audelà de son caractère figé, est sa mise en circulation dans le discours; c'est en cela qu'elle est et devient « objet partagé du débat » (Krieg-Planque 2009:56).

19 L'étude de formule a débuté par l'analyse de sa collocation correspondante (same sex+marriage), significative dans les deux corpus ( $\mathrm{LL}=743.01^{6}$ dans le Discours-PRO et $\mathrm{LL}=\mathbf{8 8 4 . 5 1}$ dans le Discours-ANTI).

De plus, si son inscription est avant tout discursive et non pas linguistique, c'est notamment par le fait que son sens, où du moins le sens qui lui est associé, dépend principalement de son contexte d'actualisation. La composition "same sex» + " marriage », dissociée de tout contexte, peut sembler ambiguë : le projet de loi pourrait être appréhendé non pas comme la reconnaissance sociale des couples de même sexe, mais la légalisation de la pédophilie et de l'inceste, l'adjectif composé "same sex » en tant que tel n'excluant pas les relations incestueuses possibles entre parents de même sexe au sein d'une même famille. Ce flou sémantique a été exploité de façon récurrente par les opposants à la légalisation du mariage entre personnes de même sexe.

21 Par ailleurs, Alice Krieg-Planque (2009), en attribuant une valeur de re à la formule, souligne son fonctionnement en tant que référent social. La formule agit en tant que dénomination, c'est un passage obligé : elle renvoie au réel. En d'autres termes, la formule définit, donne une réalité discursive à des individus, en l'occurrence des couples dont la reconnaissance juridique n'est pas encore stabilisée; stabilité que le projet de loi cherche à instaurer. Cette référence sociale, bien évidemment, est indissociable du caractère polémique de la formule, qui prend tout son sens dans la confrontation des idéologies contradictoires en vigueur dans des discours antagonistes comme ceux présents dans les Discours-ANTI et Discours-PRO.

\section{Analyse discursive de la formule « same sex marriage »}

\section{L'intégration de la formule «same sex marriage » dans le Discours- $P R O$ : souligner la dimension relationnelle inhérente et constitutive des couples de même sexe}

L'étude des principaux phénomènes de collocation associés au lexème «marriage » a permis l'identification de plusieurs formules concurrentes de "same sex marriage », en particulier "gay marriage » et " homosexual marriage ». À ce propos, Alice Krieg-Planque insiste sur le fait suivant :

[L]es formulations concurrentes ne sont pas des "synonymes en langue » de la formule, dont par exemple on pourrait rendre compte dans une analyse sémique ou que pourraient indiquer les dictionnaires de langue, mais des séquences qui 
fonctionnent de façon effective dans les discours comme des substituts plus ou moins polémiques, et plus ou moins mutuellement exclusifs (2009 : 72-73).

En effet, comme l'indique le tableau $1^{7}$, qui récapitule les principaux phénomènes collocationnels en relation avec le lexème "marriage», des différences d'usage apparaissent dans les corpus Discours-ANTI et Discours-PRO :

Tableau 1 - Collocations - Lexème « marriage » (Discours-PRO/Discours-ANTI)

\begin{tabular}{|l|l|l|l|}
\hline Mot 1 & Mot 2 & Discours-PRO & Discours-ANTI \\
\hline \multirow{4}{*}{ Marriage } & Same Sex & 743.01 & 884.51 \\
\cline { 2 - 4 } & Gay & $(8.34)$ & 129.00 \\
\cline { 2 - 4 } & Homosexual & No Matching & $(7.94)$ \\
\cline { 2 - 4 } & Heterosexual & No Matching & 53.07 \\
\hline
\end{tabular}

Premier constat: la collocation, c'est-à-dire l'association privilégiée en discours des deux lemmes «same sex» et «marriage », est significative dans les deux corpus. Deux spécificités émergent néanmoins: tout d'abord, la collocation gay+marriage n'est statistiquement pertinente que dans le Discours-ANTI (LL=129,00). De plus, deux autres collocations sont présentes dans ce discours, et non dans le Discours-PRO: homosexual+marriage et heterosexual+marriage; seule cette dernière est significative (LL=53.07).

25 En résumé, le Discours-PRO privilégie la collocation same sex+marriage (LL=743.01), contrairement au Discours-ANTI qui utilise aussi bien same sex+marriage ( $\mathrm{LL}=884.51)$, gay+marriage ( $\mathrm{LL}=129.00)$ et heterosexual+marriage $(\mathrm{LL}=53.07)$.

26 J'ai cherché ensuite les collocations significatives du lexème "couple », répertoriées dans le tableau 2:

Tableau 2 - Collocations - Lexème « couple » (Discours-PRO/Discours-ANTI)

\begin{tabular}{|l|l|l|l|}
\hline Mot 1 & Mot 2 & Discours-PRO & Discours-ANTI \\
\hline \multirow{4}{*}{ Couple } & Same sex & 1047.32 & 970.31 \\
\cline { 2 - 4 } & Gay & 152.80 & 70.49 \\
\cline { 2 - 4 } & Straight & 95.65 & No Matching \\
\cline { 2 - 4 } & Homosexual & No Matching & 59.51 \\
\cline { 2 - 4 } & Heterosexual & No Matching & 113.26 \\
\hline
\end{tabular}

Il apparaît que la collocation same sex+couple est, comme la collocation same sex+marriage, significative à la fois dans le Discours-PRO (LL=1047.32) et dans le Discours-ANTI (LL=970.31). Il y a cependant une très claire spécialisation des autres 
collocations du lexème "couple »: le Discours-PRO privilégie la paire " gay " $(\mathrm{LL}=152.80)$ et «straight » $(\mathrm{LL}=95.65)$, contrairement au Discours-ANTI qui utilise avant tout la paire "homosexual» ( $\mathrm{LL}=59.51)$ et "heterosexual » ( $\mathrm{LL}=113.26)$. Les lexèmes "gay» / "homosexual» et "straight» / "heterosexual» sont souvent considérés comme synonymes. Une troisième analyse collocationnelle, fondée sur les lexèmes "gay", "straight", "homosexual» et "heterosexual », a permis de déceler des différences sémantiques entre ces deux paires.

Contrairement aux précédentes analyses, il n'a pas été question de comparer les différences collocationnelles de chaque lemme entre les deux corpus, mais d'opposer les deux paires entre elles, à savoir "gay» / "straight» et "homosexual» / "heterosexual». Le tableau 3 regroupe les collocations significatives de "gay» et «straight» dans le Discours-PRO et le tableau 4 regroupe celles des lexèmes " homosexual » et « heterosexual » présentes dans le Discours-ANTI. Les résultats indiquent clairement une préférence dans la nature du nom commun avec lequel chaque adjectif est associé. Ceci confirme en grande partie les précédents travaux effectués à ce sujet par Baker (2004) et Bachmann (2011), qui avaient souligné une spécification adjectivale similaire dans leurs études sur corpus de certains débats dans les différentes chambres parlementaires au Royaume-Uni.

Tableau 3 - Collocations - Lexèmes « gay » / « straight » (Discours-PRO)

\begin{tabular}{|l|l|l|}
\hline Mot 1 & Mot 2 & $L L>15.13$ \\
\hline \multirow{4}{*}{ Gay } & People & 315.25 \\
\cline { 2 - 3 } & Couples & 152.80 \\
\cline { 2 - 3 } & Straight & 97.25 \\
\cline { 2 - 3 } & Attitude & 16.85 \\
\cline { 2 - 3 } & Rights & 15.28 \\
\hline Straight & Couples & 95.75 \\
\hline
\end{tabular}

Tableau 4 - Collocations - Lexèmes « homosexual » / « heterosexual » (Discours-ANTI)

\begin{tabular}{|l|l|l|}
\hline Mot 1 & Mot 2 & $L L>15.13$ \\
\hline Homosexual & Couples & 59.61 \\
\cline { 2 - 3 } & Relationships & 53.84 \\
\cline { 2 - 3 } & Behaviour & 41.90 \\
\cline { 2 - 3 } & Men & 30.99 \\
\cline { 2 - 3 } & Lobby & 29.31 \\
\hline
\end{tabular}




\begin{tabular}{|l|l|l|}
\cline { 2 - 3 } & Activity & 26.66 \\
\cline { 2 - 3 } & Population & 18.48 \\
\cline { 2 - 3 } Heterosexual & Partners & 18.48 \\
\cline { 2 - 3 } & Relationships & 42.60 \\
\cline { 2 - 3 } & Couples & 113.26 \\
\cline { 2 - 3 } & Unions & 19.18 \\
\hline
\end{tabular}

L'exemple (1) illustre l'utilisation des lexèmes «gay» et "straight» dans le corpus Discours-PRO :

(1) Changing Attitude believes the relationships of both gay and straight people are enhanced by the kind of covenant framework in which to live and love someone else in order to achieve their maximum fulfilment and happiness in life. The name given to this covenant relationship is marriage. (Changing Attitude England - Discours-PRO) ${ }^{8}$

Dans cet exemple, les adjectifs épithètes "gay» et "straight », tous deux associés au moyen de la conjonction de coordination "and", qui établit entre eux une relation d'équivalence syntaxique et les identifie clairement comme une paire sémantique, renvoient au nom commun «people ». Ainsi, «gay » et «straight » font référence à des individus, différentiables uniquement du point de vue de leur orientation sexuelle. Cette hypothèse semble être confirmée par les autres collocations significatives du lexème "gay" dans le corpus Discours-PRO, à savoir "people» (LL=315.25) et « couples » $(\mathrm{LL}=152.80)$.

31 Par ailleurs, à l'instar de l'exemple (1), la coordination des adjectifs «gay» et «straight » ( $L L=97.27)$, très présente dans le Discours-PRO, associée à un nom référant à des individus ou à la relation entre les deux individus, a deux objectifs : tout d'abord, souligner la similarité entre les couples de même sexe et les couples de sexe opposé et indiquer que rien ne les sépare en substance si ce n'est l'orientation sexuelle. Deuxièmement, l'adjonction du nom commun "relationships", lui-même repris plus tard dans l'exemple en sa qualité synonymique du terme "marriage ", semble préciser la qualité de la relation entre personnes de sexe opposé et de même sexe : ces relations, considérées comme similaires en tout point, sont avant tout fondées sur la notion d'amour, et non pas sur l'acte sexuel uniquement, comme le montre l'analyse à venir de la paire adjectivale «homosexual » et « heterosexual».

La mise en avant des individus au sein du couple explique par ailleurs les autres phénomènes collocationnels significatifs présents dans le corpus Discours-PRO. En effet, à partir du moment où l'égalité est envisagée entre gay et straight, il paraît pertinent de lutter pour la reconnaissance de leurs droits (gay+rights / $L L=15.28$ ). De plus, s'il y a reconnaissance d'inégalité, il en découle un besoin de changer les mentalités, c'est-àdire les attitudes (gay+attitude / LL=16.85 et changing+attitude / LL=240.07), à l'égard des couples de même sexe, ce qui me semble représenter l'objectif principal de l'introduction de la formule «same sex marriage ». 
(2011: 94) avait déjà remarqué ce changement corrélé aux spécialisations lexicales suivantes : alors que «homosexual » privilégie la dimension comportementale dans ses données, "gay» souligne le côté identitaire, l'adjectif «same sex» est quant à lui employé exclusivement pour décrire les relations entre couples de même sexe. Les résultats obtenus confirment ces propos, du moins concernant la préférence de «same sex " pour décrire non pas l'identité d'un individu unique mais la relation qui se crée dans un couple. En effet, "gay» s'associe tout aussi bien à "people» (LL=315.25) qu'à «couples » $(\mathrm{LL}=152.80)$ dans mes corpus. Il est possible que la nature desdits corpus en soit la cause : son objet central reste avant tout le mariage et non pas la distinction identitaire d'un individu.

En effet, comme le montre le tableau 5, illustré à travers l'exemple (2), les collocations significatives associées à "same sex» font aussi référence aux individus et à leur union instituée, et ce dans les deux corpus :

Tableau 5 - Collocations - Lexème « same sex » (Discours-PRO/Discours-ANTI)

\begin{tabular}{|l|l|l|l|l|}
\hline & \multicolumn{2}{|l|}{ Discours-PRO } & \multicolumn{2}{l|}{ Discours-ANTI } \\
\hline Mot 1 & Mot 2 & $L L>15.13$ & Word 2 & $L L>15.13$ \\
\hline \multirow{2}{*}{ Same sex } & Couples & 267.26 & Couples & 399.89 \\
\cline { 2 - 5 } & Relationships & 37.17 & Unions & 123.05 \\
\hline
\end{tabular}

(2) The Same Sex Marriage Bill marks a historic moment for civil liberties in Britain and we commend this Government for recognising that, in a democratic, tolerant and truly free society the love and commitment shared by gay and straight couples must be given equal respect and status under the law. Protecting civil liberties means ensuring that every man and woman has the freedom to pursue personal fulfilment. For many people that means sharing their life with another safe in the knowledge that their union will be equally recognised and respected (...). We also believe that by opening marriage up to same sex couples, we can only enhance the valuable role this evolving institution has, and continues to, play in our society and our lives. (The National Council for Civil Liberties Discours-PRO) ${ }^{9}$

Cet exemple établit une corrélation entre un idéal relationnel et l'institution du mariage, dont le rôle sociétal est présenté comme primordial. Le mariage, en permettant à deux individus de partager leur vie, opère l'implémentation d'un lien qu'aucune autre institution ne semble être en mesure de créer. L'accès au mariage est présenté comme la possibilité première de l'accomplissement personnel de tout individu (« to pursue personal fulfilment »). La liberté de choix, et en particulier la liberté quant au choix ou non de se marier, considérée comme une liberté civile («civil liberties»), apparaît comme un principe égalitaire essentiel. Il est essentiel car il confirme l'égalité de tout individu face à la loi (« commitment shared by gay and straight couples must be given equal respect and status under the law») dans une société démocratique, tolérante et libre comme la société britannique ( $a$ democratic, tolerant and truly free society $»)$. 

particulière du mariage, où ce dernier est perçu comme un lien entre deux individus.

\section{Éviter l'utilisation de la formule "same sex marriage » dans le Discours-ANTI : réduire les relations entre personnes de même sexe à un acte sexuel dénué de tout lien relationnel}

Les analyses précédentes ont montré que la paire "gay» / "straight» fait avant tout référence soit aux individus, soit à la relation qui les unit. Il en est de même concernant la formule "same sex marriage». Il s'agit maintenant de s'intéresser à la paire « homosexual » / " heterosexual », qui fait référence quant à elle à une dimension que je qualifierais plutôt de comportementale.

Contrairement au Discours-PRO, où «gay » et «straight » partageaient globalement les mêmes schémas collocationnels, une démarcation très nette a lieu entre "heterosexual " et « homosexual » dans le Discours-ANTI. Bien que ces deux adjectifs partagent certaines similitudes collocationnelles - comme leurs associations avec les lexèmes «couples » et "relationships" - "homosexual " a la particularité d'être associé très fréquemment à «behaviour » $(\mathrm{LL}=41.90)$ et «activity» $(\mathrm{LL}=26.66)$. Cette particularité ne se limite pas à l'emploi adjectival «homosexual». En effet, comme l'illustre l'exemple (3), le nom commun « homosexuality » est lui aussi empreint de cette dimension comportementale :

(3) There is unanimous agreement in all Islamic schools of thought that homosexuality is a forbidden act and displeases God (...). In Islam, Sodomy or homosexuality is regarded as one of the Major Sins. This is clearly recorded in the Qur'an, as well as in the teachings of Prophet Muhammad and the divinely-chosen Imams. In the Islamic legal system, homosexuality is a heinous crime and can be severely punished. The Qur'an describes the act of Sodomy as indecent (...). In addition to the legal prohibition of homosexuality, Islam poses strong ethical arguments against this act. (World Federation of KSIMC - Discours-ANTI) ${ }^{10}$

Dans cet exemple, il est fait un rapprochement sans équivoque, renforcé par l'utilisation de la conjonction de coordination « or », entre homosexualité et sodomie, ce qui tend à réduire les relations entre personnes de même sexe à cet acte sexuel seulement. Ce qui apparaît très clairement ici, c'est que cette association lexicale privilégiée entre "homosexuality" et "forbidden acts " permet de définir les relations entre personnes de même sexe non pas comme des relations amoureuses, mais comme des relations sexuelles, et principalement entre deux hommes, annihilant par la même occasion l'existence des couples de femmes. En effet, les collocations contenant l'adjectif "homosexual " ne sont significatives que lors de son association à "men" $(L L=30.99)$. Il en va de même pour l'adjectif «heterosexual». Bien que ce dernier s'associe significativement avec les lexèmes "couples " ( $\mathrm{LL}=113.26)$, "relationships " $(\mathrm{LL}=42.60)$ et « unions » $(\mathrm{LL}=19.18)$ dans le Discours-ANTI, son usage sert à indiquer que la fonction principale du mariage n'est pas de permettre d'atteindre un idéal relationnel, comme dans l'exemple (2) («maximum fulfilment and happiness in life »), mais d'offrir un cadre privilégié dans l'optique de la procréation, comme illustré dans l'exemple (4):

(4) In order to ensure their continuity, societies throughout history have enshrined in law a preference for committed heterosexual relationships which can give life to, and sustain, the next generation. In the English- 
speaking world we have called these relationships "marriage". They are based on more than love; they also encompass the possibility - by natural means - of having children, thus ensuring the future of the society in question (...). There can be no comparison between heterosexual relationships which can produce children through natural means, and homosexual relationships which cannot. Therefore, the equality premise which is driving the Equal Marriage Bill is a false one. (Dr James B. Waddell Discours-ANTI ${ }^{11}$ "Marriage", qui apparaît au tout début de l'associated memorandum rédigé par James B. Waddell. Selon ses propos, ce n'est pas la relation sentimentale qui lie deux individus, mais la finalité dite " naturelle » de toute relation, à savoir la procréation afin d'assurer la reproduction et l'avenir de la société dans la succession des générations futures (" heterosexual relationships which can give life to, and sustain, the next generation»). C'est donc pour cette raison que les couples de même sexe et couples de sexe opposé seraient et resteraient différents ("There can be no comparison between heterosexual relationships which can produce children through natural means, and homosexual relationships which cannot »), et justifieraient les raisons pour lesquelles les premiers sont incompatibles avec l'idée même de mariage ("societies throughout history have enshrined in law a preference for committed heterosexual relationships »).

41 Les collocations significatives du lexème "activity» dans le Discours-ANTI sont «sexual » ( $\mathrm{LL}=36.23)$ et «homosexual » $(\mathrm{LL}=26.66)$, ce qui confirme mes précédentes hypothèses. Il existe cependant des utilisations particulières de "same sex", comme dans l'exemple (5), où « activity » n'est pas associé à « homosexual » mais à « same sex» :

(5) Will gay marriage ease the suicide rate? Denmark should provide its LGBT community with one of the lowest levels of discrimination in the world. Same-sex activity was legalized there in 1933, and since 1977, the age of consent has been $15 \mathrm{yrs}$, irrespective of orientation or gender. It was the first country to legalise same sex unions in 1989. This provided the basis for a unique study. Over a twelve-year period, it found that death in Denmark from suicide among men in same sex registered partnerships was eight times greater than among men in heterosexual marriages. (Dr Peter G. May Discours-ANTI) ${ }^{12}$

L'auteur de cette citation, qui prend appui sur l'exemple du Danemark et des diverses lois qui ont été passées au cours $\mathrm{du} \mathrm{xx}^{\mathrm{e}}$ siècle dépénalisant et décriminalisant l'homosexualité dans ce pays, associe le nom «activity» non pas avec l'adjectif «homosexual», mais avec l'adjectif "same sex». Au premier abord, ce choix peut sembler étrange, voire paradoxal. Néanmoins, j'en propose l'assimilation à un phénomène linguistique plus général, que le linguiste américain Steven Pinker a nommé, dans un article publié dans The New York Times (Pinker 1994 ${ }^{13}$ ), «euphemism treadmill »; selon Pinker ${ }^{14}$, en effet, il est très commun d'inventer de nouveaux mots pour faire référence à un concept désagréable ou très chargé émotionnellement dans l'espoir de le décharger de la connotation négative qui lui est accolée. Cependant, par effet d'euphémisme, cette même connotation négative qui avait teinté le terme initial se retrouve transférée au nouveau terme choisi.

43 En d'autres termes : il existe un terme connoté très négativement qui fait référence à un concept particulier. Afin de se débarrasser de la connotation négative, un nouveau terme est introduit en discours, qui lui n'est pas connoté négativement. Cependant, la 
charge négative du premier terme, alors supprimée, est transférée au nouveau terme qui devient lui aussi négativement connoté. J'émets ici l'hypothèse que l'auteur cherche ainsi à transférer la charge négative véhiculée par le nom "activity » à la composition adjectivale neutre «same sex", dans l'objectif de transférer l'implication non pas de relation amoureuse mais de relation sexuelle.

\section{Conclusion : rapport sexuel, relation et individualité dans les phénomènes de nomination} marriage " et "homosexual marriage", a cherché à montrer les enjeux rhétoriques et représentatifs du phénomène de nomination. Loin d'être de simples synonymes en langue, les différentes formules présentes dans le corpus d'étude ont montré à la fois leur spécialisation sémantique et discursive, mais aussi les enjeux rhétoriques et idéologiques que leurs usages impliquent. Ainsi, alors que le Discours-PRO privilégie la formule "same sex marriage", le Discours-ANTI préfère avoir recours à d'autres constructions discursives.

De cette spécificité découlent deux stratégies argumentatives différentes: alors que « same sex marriage » souligne et implique l'idée de relation corrélée à un idéal égalitaire que seule l'ouverture du mariage aux couples de même sexe peut apporter, la formule "homosexual marriage» fait plutôt référence à un rapport sexuel, en inadéquation avec la fonction primaire du mariage, qui est la procréation. Le processus de nomination se retrouve ainsi au cœur du débat autour de la légalisation du same sex marriage au Royaume-Uni, en ce qu'il cristallise à travers la notion même de formule les enjeux argumentatifs mais surtout idéologiques qui se sont opposés.

\section{BIBLIOGRAPHIE}

BAKER, Paul. 2004. «"Unnatural Acts": Discourses of homosexuality within the House of Lords debates on gay male law reform » Journal of Sociolinguistics 8 (1) : 88-106.

BAKER, Paul. 2006. Using corpora in discourse analysis. Londres : Continuum.

BENVENISTE, Émile. 1974. Problèmes de linguistique générale. Paris : Gallimard.

BOUVERET, Myriam \& SWEETSER, Eve. 2009. « Multi-frame semantics, metaphoric extensions, and grammar » Thirty-Fifth Annual Meeting of the Berkeley Linguistics Society 1 : 49-59.

EBEL, Marianne \& FIALA, Pierre. 1977. «Recherches sur les discours xénophobes » Travaux du Centre de Recherches sémiologiques, 27-28.

HALLIDAY, Michael Alexander Kirkwood \& HASAN, Ruqaiya. 1976. Cohesion in English. London : Routledge.

HOEY, Michael. 2005. Lexical Priming: A New Theory of Words and Language. London : Routledge. 
L'HÔTE, Emilie. 2014. Identity, Narrative and Metaphor - A Corpus-Based Cognitive Analysis of New Labour Discourse. London : Palgrave Macmillan.

KRIEG-PLANQUE, Alice. 2009. La Notion de " formule » en analyse du discours : cadre théorique et méthodologique. France : Presses universitaires de Franche-Comté.

PAILLARD, Michel. 2000. Lexicologie contrastive anglais-français : formation des mots et construction du sens. Besançon : Ophrys.

PINKER, Steven. 1994. « The Game of the Name» The New York Times (3 avril 1994).

TUTIN, Agnès. 2002. «Collocations régulières et irrégulières : esquisse de typologie du phénomène collocatif » Revue Française de Linguistique Appliquée 1 (7) : 7-25.

TUTIN, Agnès. 2012. « Les Collocations dans le champ sémantique des émotions : la régularité plutôt que l'idiosyncrasie ", in Meanings, texts and other exciting things : a festschrift to commemorate the $80^{\text {th }}$ anniversary of Professor Igor Alexandrovic Mel'čuk, APRESJAN, Juri et al. (éds.). Moscou : Jazyki slavanskoj kul'tury, 602-612.

STUBBS, Michael. 2001. Words and Phrases : Corpus Studies of Lexical Semantics. London : Blackwell Publishers.

\section{NOTES}

1. Site disponible à l'adresse suivante: http://services.parliament.uk/bills/2013-14/ marriagesamesexcouplesbill/documents.html (consulté pour la dernière fois le 01/02/2017).

2. La méthodologie adoptée dans cet article s'inspire des précédents travaux de L'Hôte (2014) et en particulier de son analyse cognitive du discours du New Labour.

3. Le rapport de vraisemblance (log-likelihood, $L L$, en anglais) a été choisi pour l'ensemble des tests statistiques effectués. Ce dernier permet d'indiquer, en comparant deux corpus de taille similaire, si la différence entre le nombre d'occurrences d'un même lemme dans un corpus est significative d'un point de vue statistique, c'est-à-dire non due au hasard. Seuls les résultats supérieurs ou égaux à la valeur 15.13 (la valeur de base préconisée) ont été pris en considération dans nos analyses. Pour plus d'informations sur le rapport de vraisemblance, se référer au lien suivant : http://ucrel.lancs.ac.uk/llwizard.html

4. Wmatrix est accessible à partir de l'adresse suivante : http://ucrel.lancs.ac.uk/wmatrix/

5. Il est possible de considérer cette "invisibilisation» des couples de femmes ainsi que des autres membres de la communauté LGBTQ+ dans le Discours-ANTI comme nécessaire au fonctionnement du stéréotype négatif de la relation entre personnes de même sexe qui, nous le verrons, est présentée comme résultant uniquement d'un acte sexuel. De par les objectifs fixés et les limites de taille, ce phénomène n'est pas traité dans cette étude.

6. « $L L=743.01$ » doit se comprendre de la façon suivante : la collocation same sex+marriage a obtenu un rapport de vraisemblance (LL) de 743.01. La valeur de ce dernier dépasse la valeur plancher de 15.13 fixée précédemment, indiquant que sa présence dans le corpus est statistiquement significative, c'est-à-dire non due au hasard.

7. Chacun des tableaux se divise selon un certain de nombre de colonnes. Dans le tableau 1, par exemple, la colonne intitulée « Mot 1 » contient le premier terme de la collocation recherchée. La colonne « Mot 2 » répertorie le second terme collocationnel. Les troisième et quatrième colonnes, nommées «Discours-PRO » et «Discours-ANTI ", contiennent respectivement les rapports de vraisemblances (LL) pour chaque collocation étudiée et précisent si cette dernière est significative ou pas (« No Matching»). 
8. «Pour Changing Attitudes, un contrat, qui préconise le fait qu'aimer et vivre avec quelqu'un permet d'atteindre un degré maximal d'accomplissement et de bonheur personnel, améliore les liens qui unissent les couples aussi bien homosexuels qu'hétérosexuels. Ce contrat a un nom, celui de mariage. » (Je traduis.)

9. «Le Same Sex Marriage Act représente un moment historique dans le domaine des libertés individuelles en Grande-Bretagne et nous félicitons ce gouvernement d'avoir su reconnaître qu'au sein d'une société vraiment libre, tolérante et démocratique, l'amour et l'engagement partagés aussi bien dans des couples homosexuels qu'hétérosexuels doivent jouir d'un respect et d'un statut égaux aux yeux de la loi. La protection des libertés civiles revient à s'assurer que chaque homme et chaque femme est libre de poursuivre sa quête d'accomplissement personnel. Pour de nombreuses personnes, cela signifie pouvoir partager sa vie avec une autre personne tout en ayant l'assurance que leur union sera reconnue et respectée de la même façon (...). Nous pensons également qu'en permettant à des couples de même sexe de se marier, nous ne pouvons que renforcer le précieux rôle que cette institution en perpétuelle évolution a joué et continue de jouer dans notre société et dans nos vies. » (Je traduis.)

10. «Il existe un consensus entre les différentes écoles de pensée islamiques selon lequel l'homosexualité est un acte défendu qui déplaît à Dieu (...). Dans l'islam, la sodomie ou l'homosexualité sont considérées comme des péchés capitaux. Cela est clairement inscrit dans le Coran, ainsi que dans les enseignements du Prophète Mahomet et des imams choisis par Dieu. Dans le système législatif islamique, l'homosexualité est un crime odieux et peut être sévèrement sanctionnée. Le Coran présente la sodomie comme un acte indécent (...). Outre l'interdiction légale de l'homosexualité, l'islam oppose des arguments éthiques fermes à cet acte. » (Je traduis.)

11. "Afin d'assurer leur continuité, les sociétés ont inscrit dans leurs lois à travers l'histoire une préférence pour les relations hétérosexuelles qui permettent de mettre au monde des enfants et donc soutenir la prochaine génération. Dans le monde anglophone, nous avons nommé ces relations "mariage". Elles ne reposent pas que sur l'amour ; elles incluent aussi la possibilité - à travers des moyens naturels - d'avoir des enfants, et par conséquent d'assurer le futur de la société en question (...). Il n'y a pas de comparaison possible entre les relations hétérosexuelles, qui peuvent donner naissance à des enfants de façon naturelle, et les relations homosexuelles, qui ne le peuvent pas. Le postulat égalitaire au cœur du projet de loi Equal Marriage Bill est donc faux. » (Je traduis.)

12. «Le mariage gay parviendra-t-il à réduire le taux de suicide? Le Danemark assurait à sa communauté LGBT l'un des taux de discrimination les plus bas au monde. Les relations sexuelles entre personnes de même sexe y ont été légalisées en 1933 et, depuis 1977, l'âge de consentement est de 15 ans, peu importe l'orientation sexuelle ou le genre. C'est le premier pays à avoir légalisé les unions entre personnes de même sexe en 1989. Ceci a posé les bases pour une étude de cas unique. Étalée sur douze ans, cette dernière a montré que le taux de suicide parmi les hommes homosexuels mariés était huit fois supérieur que parmi les hommes hétérosexuels mariés. » (Je traduis.)

13. L'article en question est disponible sur le site officiel de Pinker : http://stevenpinker.com/ files/pinker/files/1994_04_03_newyorktimes.pdf (consulté le 05/05/2017).

14. Je prends appui uniquement sur la terminologie proposée par Pinker, afin d'attribuer une étiquette au phénomène présent. 


\section{RÉSUMÉS}

Cet article présente une étude approfondie de la dynamique discursive suite à l'incorporation dans le discours politique de l'expression "same sex marriage " lors des débats relatifs à l'ouverture du mariage aux couples de même sexe au Royaume-Uni en 2013. Après avoir exposé les raisons pour lesquelles cette dernière est assimilable à la notion de formule (Krieg-Planque 2009), j'expliciterai la cristallisation des enjeux sociaux qu'elle suscite, à travers une analyse à la fois qualitative et quantitative des principaux phénomènes collocationnels significatifs présents dans mes données. La comparaison systématique avec les formules concurrentes " gay marriage » et "homosexual marriage» permettra de montrer entre autres les réactions discursives, sociolinguistiques et méta-langagières que cette formule engendre, et la manière avec laquelle les représentations cognitives et sociétales de la réalité sont configurées.

This article presents a qualitative and computer-assisted, corpus-based analysis of the use of the "same sex marriage" formulae during the Marriage (Same Sex Couples) Act debates in Great Britain in 2013. By drawing on Krieg-Planque's work (2009), I want to show the reasons why same sex marriage may be assimilated to the discursive notion of formulae. The study of statistically relevant collocational patterns will help to better comprehend the ideological and argumentative dimensions of this expression. Furthermore, systematic comparisons with so-called synonymous expressions such as "gay marriage" and "homosexual marriage" will allow me to detail the sociolinguistic and metalinguistic implications at stake and question the ways cognitive representations of LGBTQ+ couples are shaped in political discourse.

\section{INDEX}

Thèmes : Recherches

Mots-clés : analyse du discours, formule, argumentation, LGBTQ, same sex marriage, RoyaumeUni

Keywords : discourse analysis, formulae, argumentation, LGBTQ, same sex marriage, United Kingdom

\section{AUTEUR}

\section{FRANÇOIS LABATUT}

Doctorant contractuel double-culture USPC, sous la direction de Mme Aliyah Morgenstern (Paris 3 Sorbonne Nouvelle) et Mme Aurélie Godet (Paris 7 Diderot), EA 4398-PRISMES.

François Labatut, diplômé d'un master en journalisme européen et certifié d'anglais, est doctorant en linguistique et civilisation américaine sous la direction de Mme Aliyah Morgenstern (Paris 3 Sorbonne Nouvelle) et Mme Aurélie Godet (Paris 7 Diderot). Ses travaux portent sur le rôle et la dimension polémique de l'expertise au niveau de la Cour suprême des États-Unis ainsi que sur les phénomènes d'argumentation et de représentation présents lors des débats autour de la légalisation du same sex marriage. 